\title{
Inhibition of sphingosine kinase 2 attenuates hypertrophic scar formation via upregulation of Smad7 in human hypertrophic scar fibroblasts
}

\author{
JIAN ZENG，BIN JIANG，XIA XIAO and ROU ZHANG \\ Department of Medical Cosmetology, The Second Affiliated Hospital, \\ University of South China, Hengyang, Hunan 421001, P.R. China
}

Received August 21, 2019; Accepted May 20, 2020

DOI: $10.3892 / \mathrm{mmr} .2020 .11313$

\begin{abstract}
The aims of the present study were to investigate the role of sphingosine kinase 2 (Sphk2) in hypertrophic scar (HS) formation and its underlying mechanisms. The expression levels of Sphk2 and Smad7 in HS tissues and healthy skin tissues of patients undergoing plastic surgery were determined using immunohistochemical staining. Subsequently, the expression levels of Sphk2 and collagen I in human embryonic skin fibroblasts (control) and human HS fibroblasts (HSF) were detected using western blot analysis and immunofluorescence assay, respectively. Following Sphk2 silencing, Smad7 overexpression or both Sphk2 and Smad7 silencing, HSF proliferative ability was assessed using Cell Counting Kit- 8 assay and proliferation-associated proteins were evaluated using western blot analysis. In addition, the level of apoptosis in HSF was assessed using flow cytometry and expression levels of apoptotic-associated proteins were determined using western blotting. Furthermore, the expression levels of collagen I and proteins in the TGF- $\beta 1 /$ Smad signaling pathway were detected using western blot analysis. The results indicated that the expression of Sphk2 was significantly increased, while Smad7 expression was decreased in HS tissue. Moreover, the upregulation of Sphk2 and collagen I expression levels was identified in HSF. The present results also indicated that Sphk2 silencing or Smad7 overexpression inhibited proliferation, but promoted apoptosis of HSF, coupled with changes in the expression levels of proliferation-associated proteins, with an increase in p21 and a decrease in cyclin D1 expression levels, and apoptosis-associated proteins, with an increase in Bax and cleaved caspase-3, and a decrease in Bcl-2, which were reversed following transfection with both Sphk2 and Smad7
\end{abstract}

Correspondence to: $\mathrm{Dr}$ Bin Jiang, Department of Medical Cosmetology, The Second Affiliated Hospital, University of South China, 35 Jiefang Road, Hengyang, Hunan 421001, P.R. China E-mail: binjianghyacin@163.com

Key words: sphingosine kinase 2, Smad7, hypertrophic scar, proliferation, apoptosis, collagen using small interfering RNA in HSF. In addition, the expression levels of transforming growth factor- $\beta 1$, phosphorylated (p)-Smad2, p-Smad3 and collagen I were reduced following Sphk2 silencing or Smad7 overexpression, which were abolished by silencing both Sphk2 and Smad7. Collectively, the present results indicated that inhibition of Sphk2 attenuated HS formation via upregulation of Smad7 expression, thus Sphk2 may serve as a potential therapeutic target for the treatment of HS.

\section{Introduction}

Hypertrophic scar (HS) is a well-known complication of skin injury, which is commonly observed after wound healing of human skin caused by burns, lacerations and surgery $(1,2)$. A previous study reported that $>90 \%$ of burn injuries and $>40 \%$ of surgical damages lead to HS, which has become a rising health problem worldwide (3). Currently, there are several therapies used to treat HS, mainly including surgery, silicone gel and laser, but no treatment has proven to be optimal, primarily due to the limited understanding of the precise underlying mechanisms of HS $(4,5)$. Therefore, it is of high importance and urgency to identify novel and viable therapies to treat HS.

Previous studies have revealed that scar formation is attributed to the abnormal proliferation and apoptotic resistance of fibroblasts, as well as excessive deposition of extracellular matrix proteins, including collagen (6-8). It has been revealed that sphingosine kinases (Sphks) catalyze the formation of sphingosine 1-phosphate, which could regulate fibrotic events in various organs, including the lungs, liver, skin and kidneys $(9,10)$. Moreover, Sphks are ubiquitously expressed and Sphk2 is one of the most common subtypes (11). A previous study reported that silencing of Sphk 2 could increase the proliferation of renal mouse mesangial cells and fibroblasts (12). Emerging evidence also supports the hypothesis that Sphk2 deficiency could inhibit collagen expression and attenuate unilateral ureteral obstruction-induced mouse kidney fibrosis by enhancing the expression of Smad7 (13). In addition, the Sphk2 inhibitor, ABC294640, was reported to suppress proliferation and promote apoptosis in a human skin squamous cell carcinoma cell line (14). Furthermore, 
it has been revealed that inhibition of Sphk2 can alleviate psoriasis-like skin disease (15). However, the effect of Sphk2 in HS formation remains unknown.

Therefore, the present study investigated the effect of Sphk2 in scar formation and its underlying regulatory mechanisms in human HS fibroblasts (HSF).

\section{Materials and methods}

Tissue samples. A total of 20 paired HS tissues (HS group) and adjacent healthy skin tissues (healthy group) were collected from 20 patients who underwent plastic surgery from February 2017 to March 2018 at The Second Affiliated Hospital, University of South China (Table I). The size of the tissue was $1 \times 1 \times 1 \mathrm{~cm}$. The ages of all the patients ranged from 19-50 years. The specimens were selected according to the following criteria: i) Skin or HS tissue specimens were identified by clinicians, which was in accordance with a previous study (16); ii) patients with pituitary diseases, adrenal diseases, infectious diseases, skin diseases, local infections and ulcers were excluded from the study; and iii) patients with scars who had undergone previous treatments were excluded from the study (17). All tissues were immediately immerged in liquid nitrogen $\left(-196^{\circ} \mathrm{C}\right)$ after surgery until further use. The present study was approved by the Ethics Committee of The Second Affiliated Hospital, University of South China. Written informed consent was provided from each patient or their legal guardians.

Cell culture. Human HSF were purchased from Shanghai Guandao Biological Engineering Co., Ltd. (cat. no. c0618) and human embryonic skin fibroblasts, CCC-ESF-1 (control), were purchased from the Shanghai Zibo Biological Technology Co., Ltd. (cat no. YB-aTcc-3084). Cells were cultured in RPMI-1640 medium (Gibco; Thermo Fisher Scientific, Inc.) containing 10\% FBS (Gibco; Thermo Fisher Scientific, Inc.) and $1 \%$ ascorbic acid (Sigma-Aldrich; Merck KGaA). All cells were incubated at $37^{\circ} \mathrm{C}$ in a humidified incubator with $5 \% \mathrm{CO}_{2}$.

Cell transfection. Prior to cell transfection, cells were seeded into 6-well plates $\left(1 \times 10^{6}\right.$ cells/well) and incubated at $37^{\circ} \mathrm{C}$ for $24 \mathrm{~h}$ to reach $80 \%$ confluency. Subsequently, $100 \mathrm{nmol} / \mathrm{l}$ small interfering (si)RNA-Sphk2 (5'-CAGGATTGCGCTCGCTTT CAT-3'), the negative control (NC; Sphk2-NC; 5'-UUCUCC GAACGUGUCACGUTT-3'), siRNA-Smad7 (5'-GGCTGG AGGTCATCTTCAA-3'), Smad7-NC (5'-AATTGTCCGAAC GTGTCACGT-3'), pcDNA3.1-Smad7 or empty pcDNA3.1 vector (pcDNA-NC) were transfected into HSF, which were all synthesized by Shanghai GenePharma Co., Ltd.. To perform the cell transfection experiments, Lipofectamine ${ }^{\circledR} 2000$ (Invitrogen; Thermo Fisher Scientific, Inc.) was used following the manufacturer's instructions. Following $48 \mathrm{~h}$ of transfection at $37^{\circ} \mathrm{C}$, the cells were collected for subsequent experimentation.

Immunohistochemical staining. The tissues were fixed with $10 \%$ formalin at room temperature for $24 \mathrm{~h}$ and then embedded in paraffin at $62^{\circ} \mathrm{C}$ for $45 \mathrm{~min}$. Paraffin-embedded specimens were cut into $4-\mu \mathrm{m}$ thick sections, deparaffinized and rehydrated with a graded ethanol and xylene series at room temperature. Following blocking with $10 \%$ normal goat serum (Wuhan Servicebio Technology Co., Ltd.) for $10 \mathrm{~min}$ at $37^{\circ} \mathrm{C}$, slides were incubated overnight $(12 \mathrm{~h})$ at $4^{\circ} \mathrm{C}$ with the following primary antibodies: Anti-Sphk2 (ProteinTech Group, Inc.; cat. no. 17096-1-AP; 1:100) and anti-Smad7 antibody (Santa Cruz Biotechnology, Inc.; cat. no. sc-101152; 1:1,000). Slides were then incubated with horseradish peroxidase (HRP)-secondary antibodies (Abcam; cat. nos. ab6721 or ab6728; $1: 1,000)$ for $2 \mathrm{~h}$ at room temperature, stained with diaminobenzidine (Beyotime Institute of Biotechnology) at room temperature for $5 \mathrm{~min}$, and counterstained with $0.2 \%$ hematoxylin at room temperature for $1 \mathrm{~min}$. A light microscope (magnification, x200; Carl Zeiss AG) was used to analyze the degree of staining for each image. Brown cellular staining was considered to indicate positive protein expression (18).

Immunofluorescence assay. Cells were washed with PBS, fixed with $4 \%$ paraformaldehyde for $30 \mathrm{~min}$ at room temperature, permeabilized with $0.1 \%$ Triton X-100 and blocked with 5\% BSA (Sigma-Aldrich; Merck KGaA) for $1 \mathrm{~h}$ at room temperature. Cells were then incubated with an anti-collagen I primary antibody (Abcam; cat. no. ab34710; 1:1,000) overnight at $4^{\circ} \mathrm{C}$. After washing with PBS, cells were immersed in fluorescein isothiocyanate-conjugated goat anti-rabbit secondary antibody (Boster Biological Technology; cat. no. BA1105; $1: 10,000)$ at $37^{\circ} \mathrm{C}$ for $1 \mathrm{~h}$. Nuclei were stained with DAPI (Roche Diagnostics) in the dark at room temperature for $5 \mathrm{~min}$, and a fluorescence microscope (magnification, x200; Nikon Corporation) was used to obtain fluorescence images.

Cell proliferation assay. A Cell Counting Kit-8 assay (CCK-8; Shanghai Yi Sheng Biotechnology Co., Ltd.) was used to analyze the ability of cell proliferation, according to the manufacturer's instructions. At $48 \mathrm{~h}$ after transfection, cells were seeded in 96-well plates ( $1 \times 10^{4}$ cells/well). At 24,48 and $72 \mathrm{~h}, 10 \mu \mathrm{l} \mathrm{CCK}-8$ solution was added to each well. Following incubation at $37^{\circ} \mathrm{C}$ for $1 \mathrm{~h}$, the optical density was measured at $450 \mathrm{~nm}$ on a microplate reader.

Cell apoptosis assay. Following transfection for $48 \mathrm{~h}, \mathrm{HSF}$ were subjected to an apoptosis assay. $\operatorname{HSF}\left(8 \times 10^{5}\right)$ were stained with Annexin V-PE/7AAD for $15 \mathrm{~min}$ at room temperature using a cell apoptosis detection kit (Nanjing KeyGen Biotech Co., Ltd.) according to the manufacturer's instructions. Then, cell apoptosis was analyzed using a BD FACSVerse ${ }^{\mathrm{TM}}$ flow cytometer (BD Biosciences). Subsequently, the data were analyzed using FlowJo software (version 10; FlowJo LLC).

Reverse transcription-quantitative PCR (RT-qPCR). Total RNA was extracted from cells using TRIzol ${ }^{\circledR}$ reagent (Invitrogen; Thermo Fisher Scientific, Inc.). First-strand cDNA synthesis was conducted using a Sensiscript RT kit (Takara Biotechnology Co., Ltd.) according to the manufacturer's protocol at $37^{\circ} \mathrm{C}$ for $15 \mathrm{~min}$ and $85^{\circ} \mathrm{C}$ for $5 \mathrm{sec}$. qPCR was performed using iTaq ${ }^{\mathrm{TM}}$ Universal SYBR ${ }^{\circledR}$ Green Supermix (Bio-Rad Laboratories, Inc.) on an ABI 7500 system (Applied Biosystems; Thermo Fisher Scientific, Inc.). The following 
Table I. Profile of each sample from each volunteer.

\begin{tabular}{rlrlr}
\hline Sample & Sex & Age, years & Localization & $\begin{array}{c}\text { Time after trauma } \\
\text { or burn, months }\end{array}$ \\
\hline 1 & Female & 19 & Shoulder & 7 \\
2 & Female & 21 & Chest & 10 \\
3 & Male & 28 & Chest & 11 \\
4 & Female & 39 & Back & 10 \\
5 & Male & 50 & Shoulder & 8 \\
6 & Male & 33 & Back & 9 \\
7 & Male & 25 & Ear & 12 \\
8 & Female & 31 & Trunk & 10 \\
9 & Female & 44 & Forehead & 6 \\
10 & Female & 38 & Chest & 11 \\
11 & Male & 26 & Lower leg & 8 \\
12 & Male & Back & 12 \\
13 & Male & Female & Shoulder & 7 \\
14 & Female & 27 & Forehead & 8 \\
15 & Male & 29 & Shoulder & 7 \\
16 & Male & 37 & Nose & 7 \\
17 & Female & 49 & Back & 12 \\
18 & Female & 20 & Lower leg & 9 \\
19 & Male & 32 & Shoulder & 9 \\
20 & & 23 & Chest & 11
\end{tabular}

thermocycling conditions were used: Initial denaturation at $95^{\circ} \mathrm{C}$ for $7 \mathrm{~min}$; and 40 cycles of $95^{\circ} \mathrm{C}$ for $15 \mathrm{sec}$ and $60^{\circ} \mathrm{C}$ for $30 \mathrm{sec}$; and a final extension at $72^{\circ} \mathrm{C}$ for $30 \mathrm{sec}$. The following primers were used: Sphk2 forward, 5'-CCAGTGTTGGAG AGCTGAAGGT-3' and reverse, 5'-GTCCATTCATCTGCT GGTCCTC-3'; Smad7 forward, 5'-GCTATTCCAGAAGAT GCTGTTC-3' and reverse, 5'-GTTGCTGAGCTGTTCTGA TTTG-3'; and GAPDH forward, 5'-GGAGCGAGATCCCTC CAAAAT-3', and reverse 5'-GGCTGTTGTCATACTTCT CATGG-3'. The $2^{-\Delta \Delta \mathrm{Cq}}$ method was used for data analysis with normalization to GAPDH (19).

Western blot analysis. Proteins in tissues and cells were extracted using protein lysis buffer (RIPA; Beyotime Institute of Biotechnology) and the concentration was determined using a bicinchoninic acid assay protein assay kit (Beyotime Institute of Biotechnology). Equal amounts of protein $(40 \mu \mathrm{g}$ per lane) were loaded on $10 \%$ SDS-PAGE and transferred onto a PVDF membrane (EMD Millipore). The membranes were subsequently blocked with $5 \%$ skimmed milk for $1 \mathrm{~h}$ at room temperature and incubated with primary antibodies overnight at $4^{\circ} \mathrm{C}$. After washing three times with TBS- $0.2 \%$ Tween-20, membranes were probed with a goat anti-rabbit HRP-conjugated secondary antibody (Cell Signaling Technology, Inc.; cat. no. 7074S; 1:3,000) or horse anti-mouse HRP-conjugated secondary antibody (Cell Signaling Technology, Inc.; cat. no. 7076S; 1:3,000) at room temperature for $1 \mathrm{~h}$. Proteins were visualized with an enhanced chemiluminescence detection system (Applygen Technologies, Inc.) and subsequently quantified using ImageJ software (version $1.52 \mathrm{r}$,
National Institutes of Health). The protein expression of the bands was normalized against the gray value of GAPDH. Anti-Smad7 antibody (cat. no. sc-101152; 1:1,000) was purchased from Santa Cruz Biotechnology, Inc. Anti-collagen I antibody (cat. no. ab34710; 1:1,000) was purchased from Abcam. Anti-Sphk2 (cat. no. 32346S; 1:1,000), anti-cyclin D1 (cat. no. 55506T; 1:1,000), anti-p21 (cat. no. 2947T; 1:1,000), anti-Bax (cat. no. 5023T; 1:1,000), anti-Bcl-2 (cat. no. 4223T; 1:1,000), anti-cleaved caspase-3 (cat. no. 9661T; 1:1,000), anti-caspase-3 (cat. no. 14220T; 1:1,000), anti-transforming growth factor (TGF)- $\beta 1$ (cat. no. 3709S; 1:1,000), anti-phosphorylated (p)-Smad2 (cat. no. 18338T; 1:1,000), anti-p-Smad3 (cat. no. 9520T; 1:1,000), anti-Smad2 (cat. no. 5339T; 1:1,000), anti-Smad3 (cat. no. 9523T; 1:1,000) and anti-GAPDH (cat. no. 5174T; 1:1,000) antibodies were obtained from Cell Signaling Technology, Inc.

Statistical analysis. All results were obtained from $\geq 3$ independent experiments and all data were analyzed using SPSS 20.0 software (SPSS, Inc.). Data are presented as the mean \pm SD. Quantitative data between two groups was analyzed using an unpaired Student's t-test, and the comparisons among multiple groups were conducted using a one-way ANOVA followed by Tukey's post hoc test. $\mathrm{P}<0.05$ was considered to indicate a statistically significant difference.

\section{Results}

Sphk2 is upregulated and Smad7 is downregulated in HS tissues and HSF. To investigate the effect of Sphk2 and Smad7 

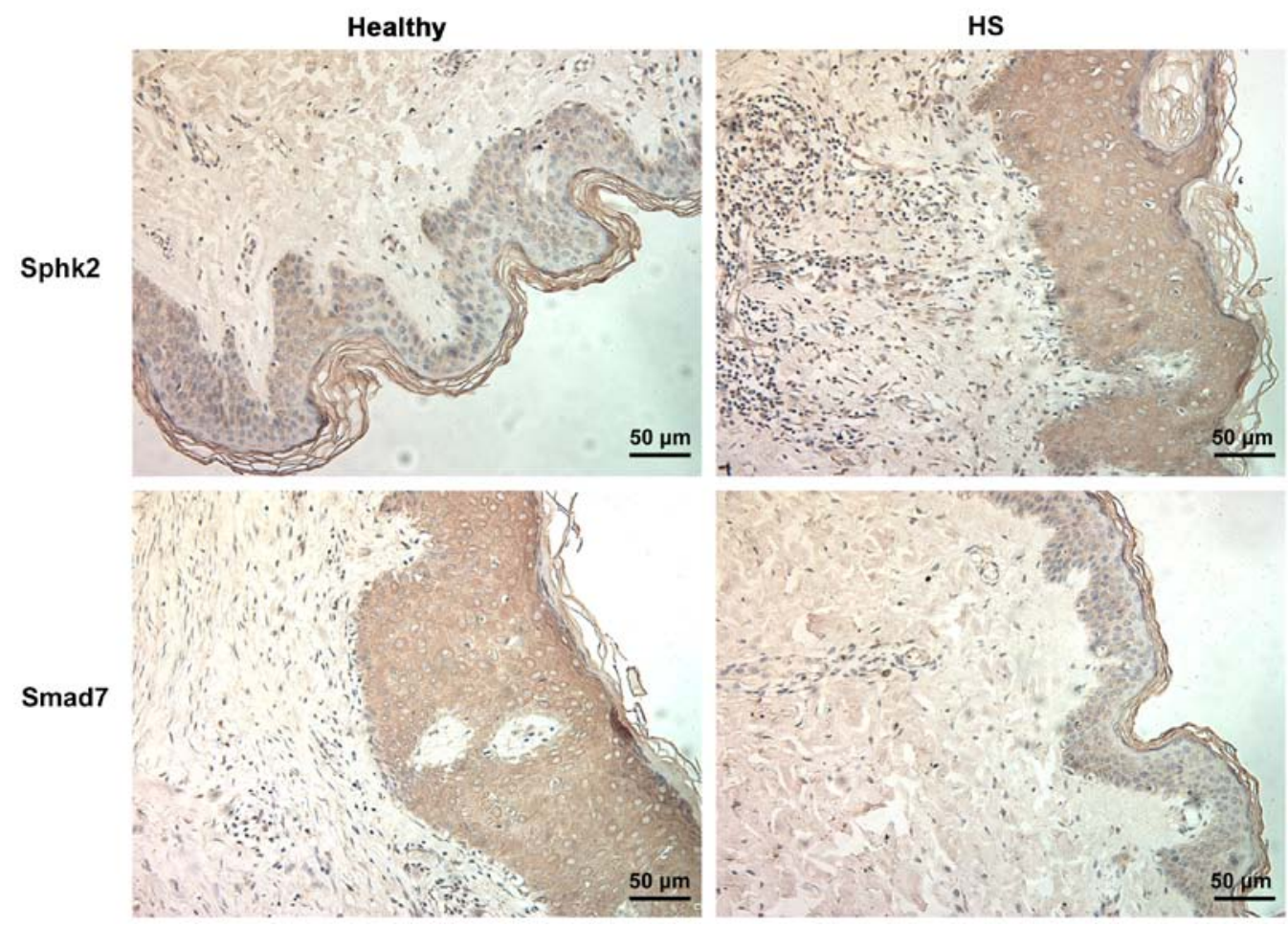

Figure 1. Expression of Sphk2 is increased, whereas Smad7 is decreased in HS tissue compared with that in adjacent healthy skin tissues. Representative immunohistochemical staining for Sphk2 and Smad7 in healthy skin and HS tissues. Scale bar, $50 \mu \mathrm{m}$. Sphk2, sphingosine kinase 2; HS, hypertrophic scar.

in scar formation, HS tissues and adjacent healthy skin tissues were obtained to assess the expression levels Sphk2 and Smad7. It was determined that the expression of Sphk2 was increased in the HS group compared with the healthy group (Fig. 1). In addition, Smad7 expression was downregulated in HS tissues.

To further assess the possible mechanisms of Sphk2 in scar formation, HSF and CCC-ESF-1 cells were used in the subsequent experiments. The results indicated that the protein and mRNA expression levels of Sphk2, detected by western blot analysis and RT-qPCR, respectively, were significantly increased in the HSF group (Fig. 2A and B). In addition, the immunofluorescence assay results revealed that the protein expression of collagen I was increased in HSF compared with the control group. Therefore, the results demonstrated that Sphk2 was upregulated, whereas Smad7 was downregulated, in HS tissues and HSF, thus suggesting a potential regulatory effect between Sphk2 and Smad7.

Sphk2 silencing or Smad7 overexpression is successfully established in HSF. Sphk2-siRNA or Smad7-pcDNA were transfected into HSF, and the transfection efficiency was determined using western blot analysis and RT-qPCR. It was demonstrated that Sphk2-siRNA transfection significantly downregulated the expression of Sphk2 (Fig. 3A and B). Moreover, the expression of Smad7 was significantly upregulated in HSF transfected with Smad7-pcDNA compared with the pcDNA-NC group (Fig. 3C and D). Thus, the results indicated that Sphk2 silencing or Smad7 overexpression were successfully established.
Sphk2 silencing inhibits proliferation of HSF via upregulation of Smad7 expression. The expression of Smad7 was examined by RT-qPCR after transfection with Smad7-siRNA, and it was revealed that the expression of Smad7 was significantly decreased in the Smad7-siRNA group compared with the Smad7-NC group (Fig. 4A). To examine the effect of Sphk2 silencing on proliferation in HSF, a CCK-8 kit was used to determine cell proliferation ability. It was determined that Sphk2 silencing inhibited the proliferation of HSF compared with the Sphk2-NC group, and that Smad7 overexpression also inhibited cell proliferation; however, this suppression of proliferation was reversed following transfection with Sphk2-siRNA and Smad7-siRNA (Fig. 4B). Moreover, the expression levels of the proliferation-associated proteins were evaluated by western blot analysis. It was demonstrated that Sphk2 silencing decreased the expression of cyclin D1, accompanied by a significant increase in p21 expression compared with the Sphk2-NC group (Fig. 4C and D). The overexpression of Smad7 demonstrated the same results as Sphk2 silencing on the expression levels of the proliferation-associated proteins. However, cells transfected with both Sphk2-siRNA and Smad7-siRNA had increased expression of cyclin D1 and decreased expression of p21 compared with cells transfected with Smad7-siRNA alone. Therefore, these results indicated that Sphk2 silencing inhibited the proliferation of HSF by upregulating Smad7.

Sphk2 silencing promotes apoptosis of HSF via upregulation of Smad7 expression. To evaluate the effect of Sphk2 silencing on apoptosis of HSF, flow cytometry was performed, and it was demonstrated that the number of apoptotic cells 

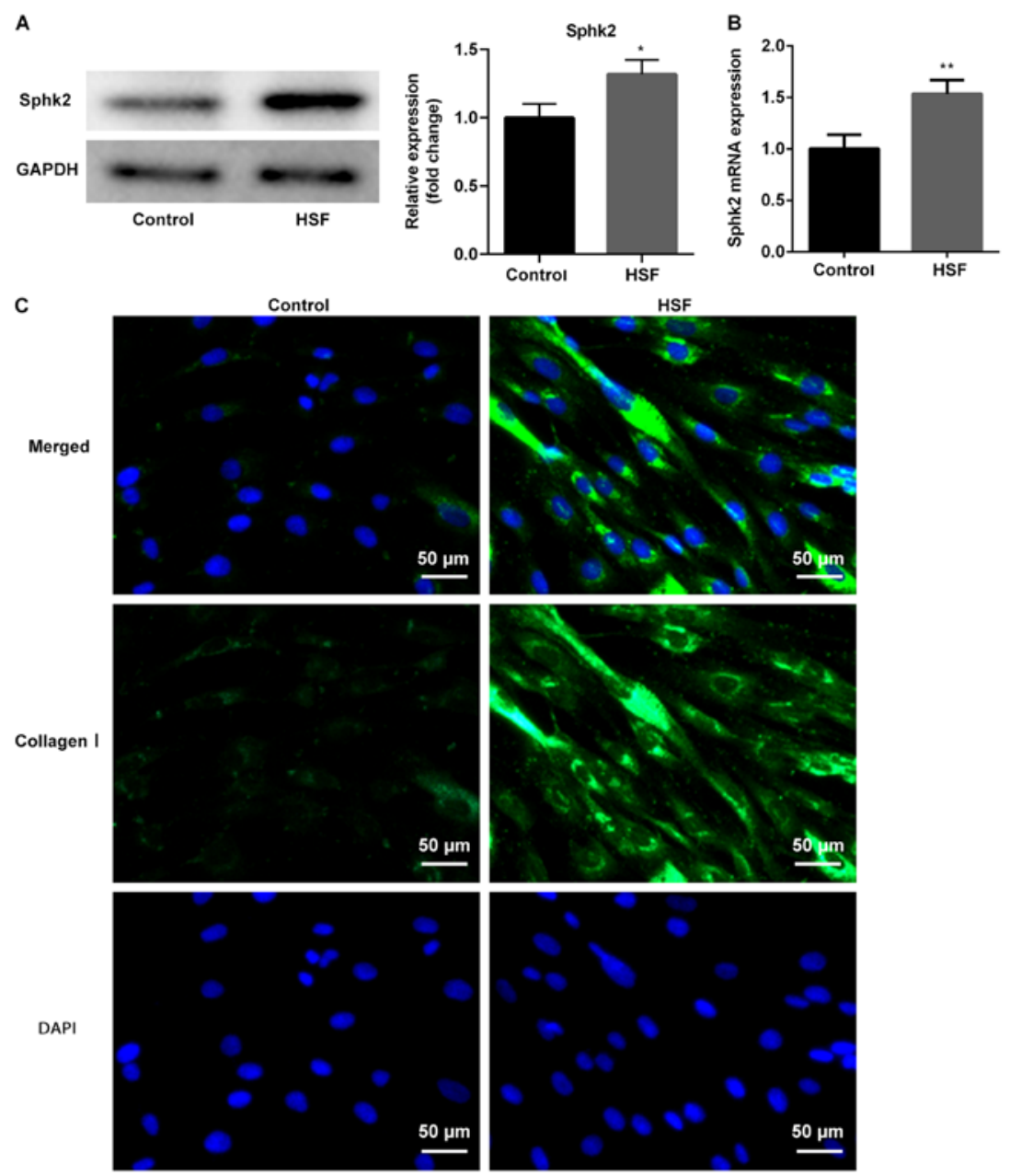

Figure 2. Expression levels of Sphk2 and collagen I are upregulated in HSF compared with the control group. (A) Western blotting and (B) reverse transcription-quantitative PCR were used to assess the protein and mRNA expression levels of Sphk2, respectively. (C) Expression of collagen I was detected using immunofluorescence assay. Scale bar, $50 \mu \mathrm{m}$. All experiments were repeated three times independently. Data are presented as the mean \pm SD. "P $<0.05$, ${ }^{* *} \mathrm{P}<0.01$ vs. the control. Sphk2, sphingosine kinase 2; HSF, hypertrophic scar fibroblasts.

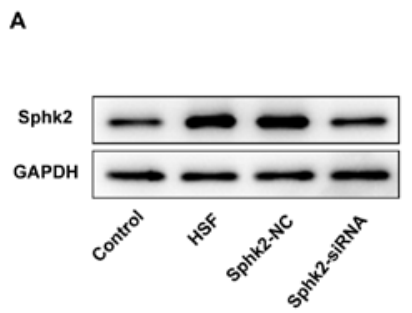

C

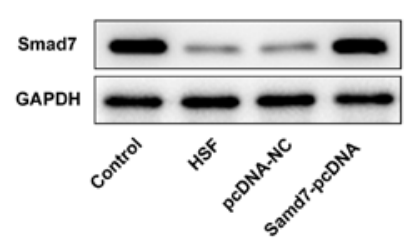

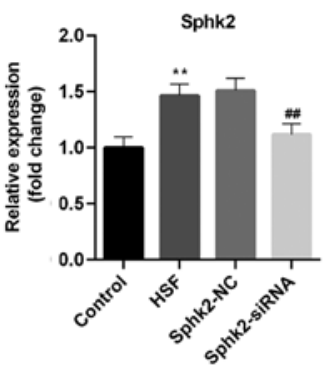

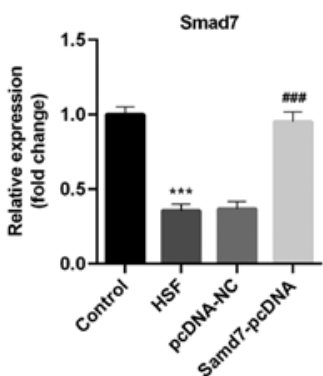

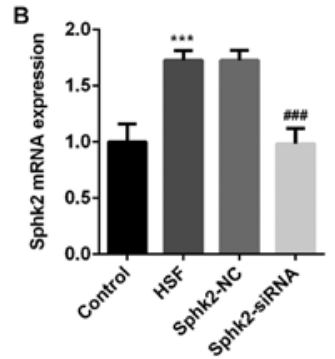

D

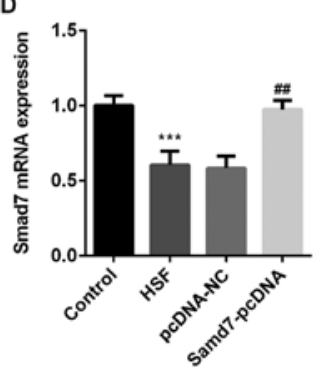

Figure 3. Sphk2-siRNA or Smad7-pcDNA3.1 transfection into HSF. (A) Western blot analysis and (B) RT-qPCR were used to evaluate the protein and mRNA expression levels of Sphk2 after transfection with or without Sphk2, respectively. ${ }^{* *} \mathrm{P}<0.01,{ }^{* * *} \mathrm{P}<0.001$ vs. the control; ${ }^{\# \#} \mathrm{P}<0.01$, ${ }^{\# \#} \mathrm{P}<0.001 \mathrm{vs}$. Sphk2-NC. Protein and mRNA expression levels of Smad7 were detected by (C) western blot analysis and (D) RT-qPCR, respectively. ${ }^{* * *} \mathrm{P}<0.001$ vs. the control; ${ }^{\# \#} \mathrm{P}<0.01$,

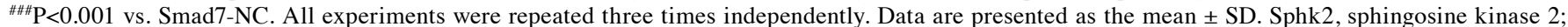
NC, negative control; siRNA, small interfering RNA; RT-qPCR, reverse transcription-quantitative PCR; HSF, hypertrophic scar fibroblasts. 

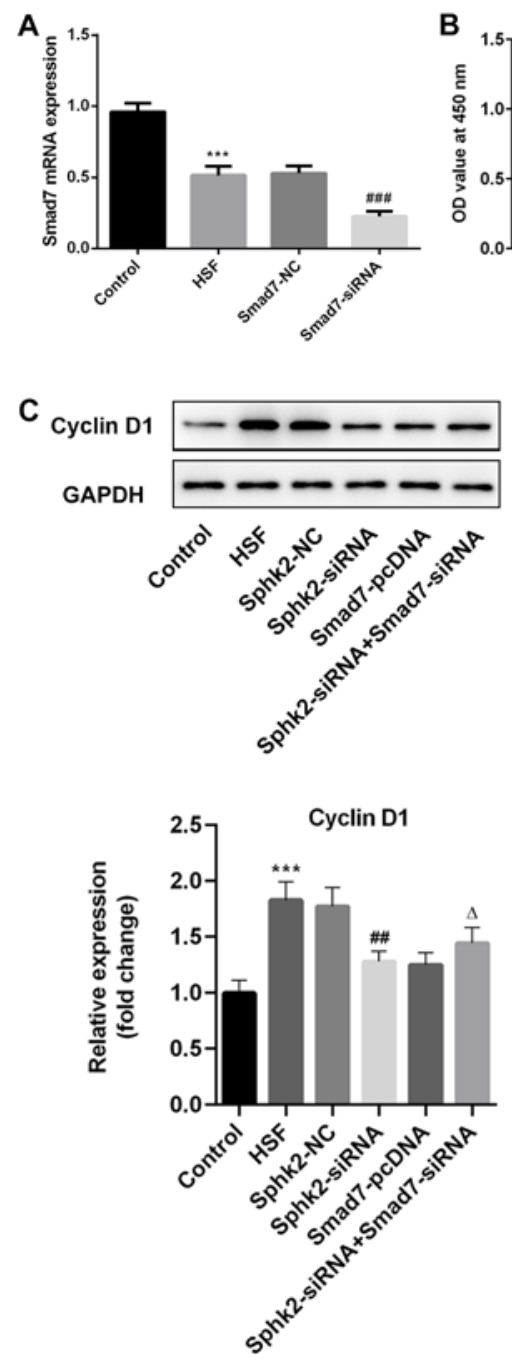

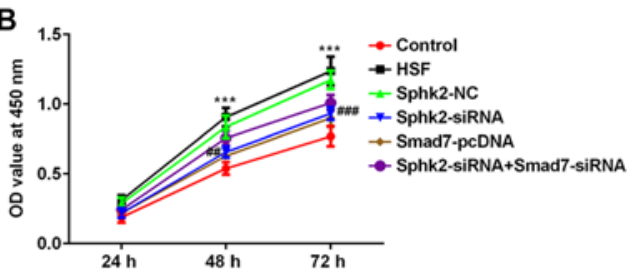

D
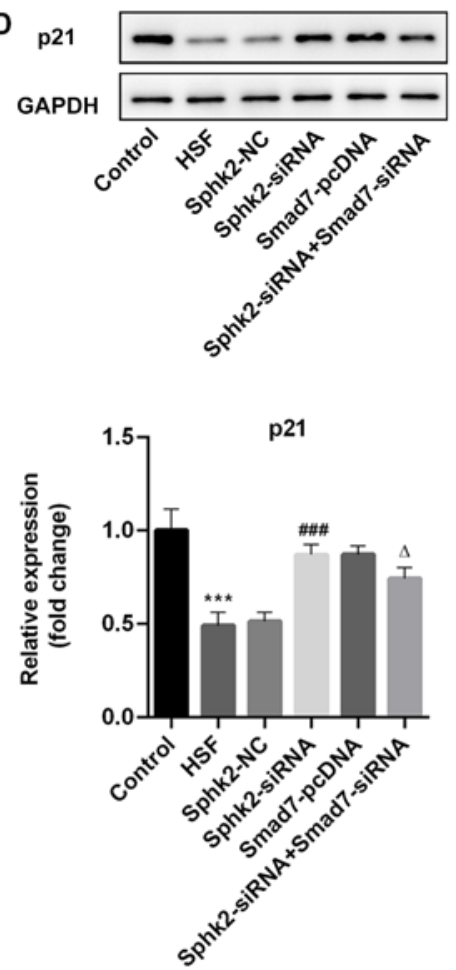

Figure 4. Effect of Sphk2-siRNA, Smad7-pcDNA and Sphk2-siRNA + Smad7-siRNA on the proliferation of HSF. (A) Expression of Smad7 was assessed using RT-qPCR after transfection. ${ }^{* * *} \mathrm{P}<0.001$ vs. the control; ${ }^{\# \# "} \mathrm{P}<0.001$ vs. Smad7-NC. (B) Cell proliferation was assessed using a Cell Counting Kit-8 assay. Expression levels of proliferation-associated proteins (C) cyclin D1 and (D) p21 were determined using western blot analysis. ${ }^{* * * *} \mathrm{P}<0.001 \mathrm{vs.}$ the control; ${ }^{\# \#} \mathrm{P}<0.01,{ }^{\# \# \prime} \mathrm{P}<0.001$ vs. Sphk2-NC; ${ }^{\wedge} \mathrm{P}<0.05$ vs. Sphk2-siRNA. All experiments were repeated three times independently. Data are presented as the mean $\pm \mathrm{SD}$. Sphk2, sphingosine kinase 2; siRNA, small interfering RNA; NC, negative control; HSF, hypertrophic scar fibroblasts; OD, optical density.

was significantly decreased in the HSF group compared with the control group (Fig. 5A and B). After transfection with Sphk2-siRNA or Smad7-pcDNA, the ratio of cell apoptosis was enhanced compared with the untreated cells. However, transfection with both Sphk2-siRNA and Smad7-siRNA reversed the increase in the number of apoptotic cells.

The expression levels of apoptosis-associated proteins were subsequently investigated. It was revealed that Sphk2-siRNA significantly upregulated the expression levels of pro-apoptotic proteins, Bax and cleaved caspase-3, whereas the expression of the anti-apoptotic protein Bcl-2 was significantly downregulated compared with the Sphk2-NC group (Fig. 5C). Similar results were obtained with Smad7-pcDNA, with the expression levels of the aforementioned apoptosis-related proteins demonstrating the same trend. By contrast, the transfection with both Sphk2-siRNA and Smad7-siRNA partially reversed the effects of Sphk2-siRNA alone on the expression levels of apoptotic proteins. Collectively, the results indicated that Sphk2 silencing promoted apoptosis of HSF via upregulation of Smad7.
Sphk2 silencing inactivates TGF- $\beta 1 /$ Smad signaling and collagen I expression in HSF via upregulation of Smad7 expression. To examine the regulatory mechanisms of Sphk2 on scar formation, western blot analysis was performed to assess the expression levels of TGF- $\beta 1 /$ Smad signaling proteins and collagen I. The results revealed that Sphk2 silencing or Smad7 overexpression alone decreased the expression levels of TGF- $\beta 1$, p-Smad2, p-Smad3 and collagen I, while cells transfected with both Sphk2- and Smad7-siRNA had increased expression levels of the aforementioned proteins compared with cells transfected with Sphk2-siRNA and Smad7-pcDNA alone (Fig. 6A and B). Therefore, the results indicated that Sphk 2 silencing inhibited TGF- $\beta 1 /$ Smad signaling and collagen I expression in HSF via upregulation of Smad7.

\section{Discussion}

HS is a fibrotic disease characterized by the over-proliferation and activation of fibroblasts, which is often considered as a benign skin tumor (20). The role of Sphk2 in skin diseases 
A
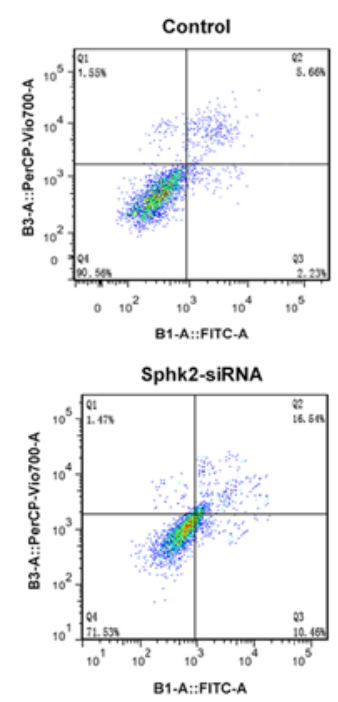

C

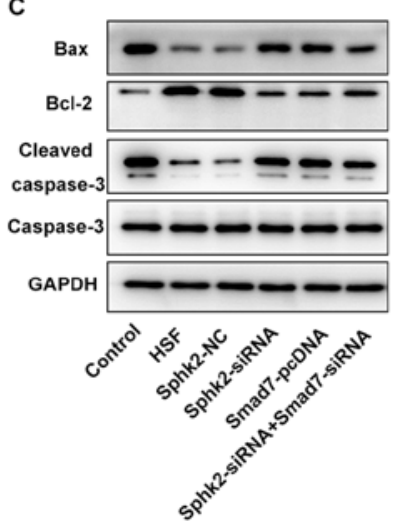

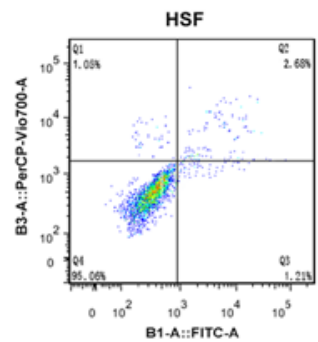

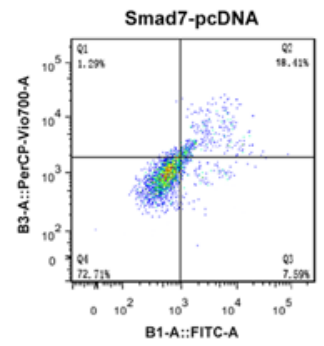

Bax

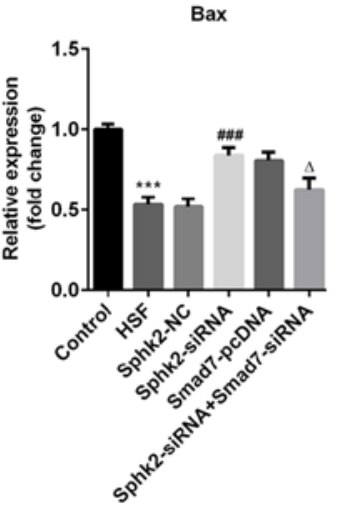

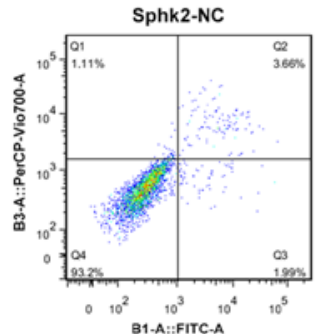

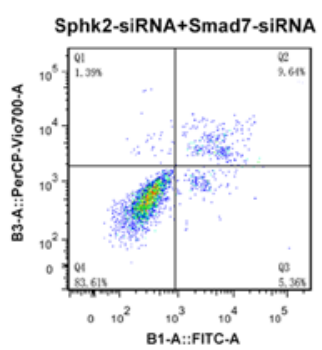

Bcl-2

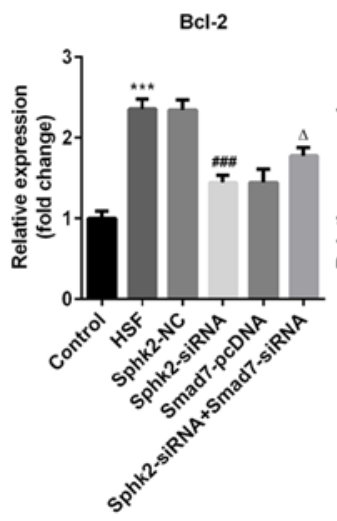

B

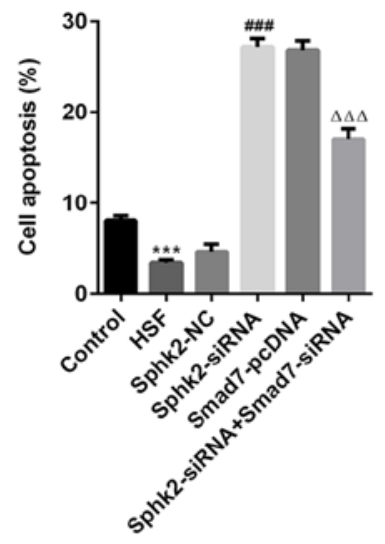

Figure 5. Effect of Sphk2-siRNA, Smad7-pcDNA and Sphk2-siRNA + Smad7-siRNA on apoptosis of HSF. (A) Cell apoptosis was assessed using flow cytometric analysis. (B) Quantification of flow cytometric data. (C) Expression levels of apoptosis-associated proteins Bax, Bcl-2 and cleaved caspase-3 were determined using western blot analysis. ${ }^{* * *} \mathrm{P}<0.001$ vs. the control; ${ }^{\# \# \#} \mathrm{P}<0.001$ vs. Sphk2-NC; ${ }^{\Delta \mathrm{P}}<0.05,{ }^{\Delta \Delta} \mathrm{P}<0.01,{ }^{\Delta \Delta} \mathrm{P}<0.001$ vs. Sphk2-siRNA. All experiments were repeated three times independently. Data are presented as the mean \pm SD. Sphk2, sphingosine kinase 2; siRNA, small interfering RNA; NC, negative control; HSF, hypertrophic scar fibroblasts.

has been reported in several previous studies $(14,15)$; however, to the best of our knowledge, the expression and function of Sphk2 in HS has yet to be elucidated. In the present study, it was initially found that Sphk2 was upregulated, but Smad7 was downregulated in HS tissues compared with healthy skin tissues. Furthermore, Sphk2 silencing or Smad7 overexpression inhibited proliferation, promoted apoptosis and inactivated Smad signaling and collagen expression in HSF, which were eliminated by the silencing of both Sphk2 and Smad7. Therefore, it was speculated that inhibition of Sphk2 could alleviate scar formation by upregulating Smad7 expression.

Previous studies have revealed that the abnormal and excessive proliferation of fibroblasts is one of the dominant factors in the occurrence and development of HS (21). Moreover, it has been reported that inhibition of HSF proliferation could suppress the development of HS $(22,23)$. In addition, Sphk2 silencing can reduce proliferation of various types of tumor cells, such as non-small cell lung cancer (NSCLC), papillary thyroid carcinoma, colorectal cancer and skin squamous cell carcinoma (14,24-26). It has also been revealed that the activation of Sphk2 may contribute to bile duct ligation-induced liver fibrosis and cholangiocyte proliferation (27). Therefore, the present study investigated whether Sphk2 silencing could affect the proliferation of HSF. The present results indicated that silencing of Sphk2 and the overexpression of Smad7, reduced the proliferative ability of HSF, decreased cyclin D1 expression and increased p21 expression. Furthermore, it was revealed that transfecting Sphk2-siRNA and Smad7 siRNA into HSF abrogated the reduction in cell proliferation. Collectively, these results indicated that Sphk2 silencing can inhibit proliferation of HSF via upregulation of Smad7 expression.

Previous studies have reported that apoptotic resistance of fibroblasts contributes to the development and progression of scar formation, and the induction of fibroblast apoptosis reduces HS formation $(28,29)$. Furthermore, silencing of Sphk2 was revealed to suppress cell proliferation and promote cell apoptosis in NSCLC and skin squamous cell carcinoma $(14,30)$. It has also been revealed that Sphk2 silencing suppresses the proliferation and induces the apoptosis of osteoarthritis chondrocytes (31). In the present study, HSF apoptosis was observed following transfection with Sphk2-siRNA or Smad7-pcDNA. Moreover, the expression levels of the pro-apoptosis proteins, Bax and cleaved caspase-3, were significantly upregulated, coupled with a downregulation 
A
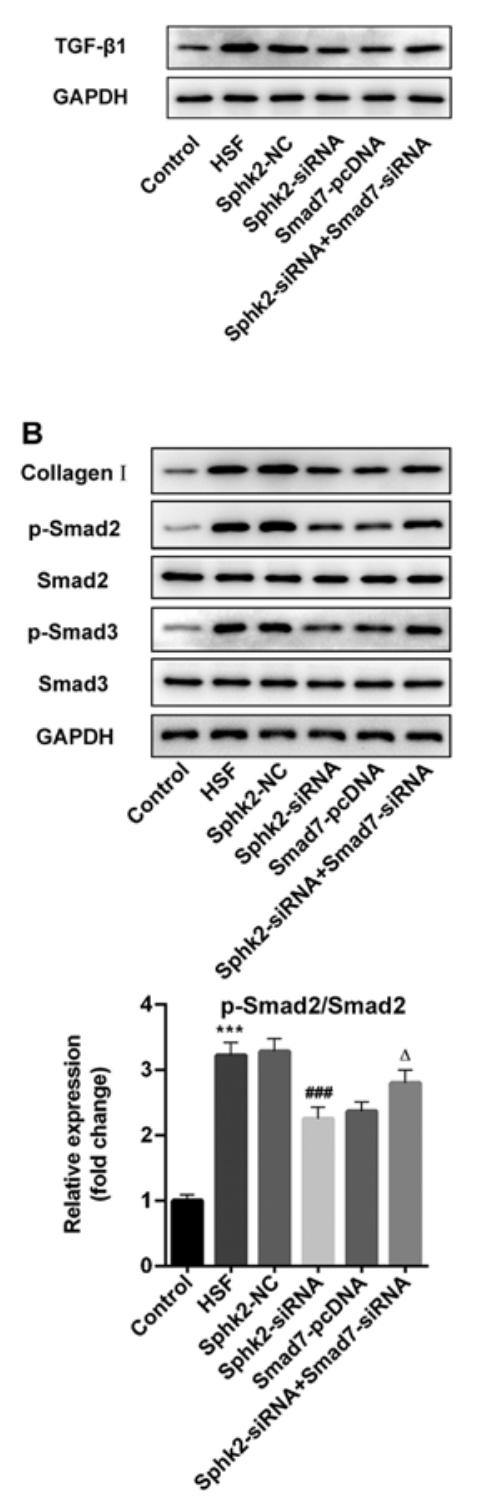
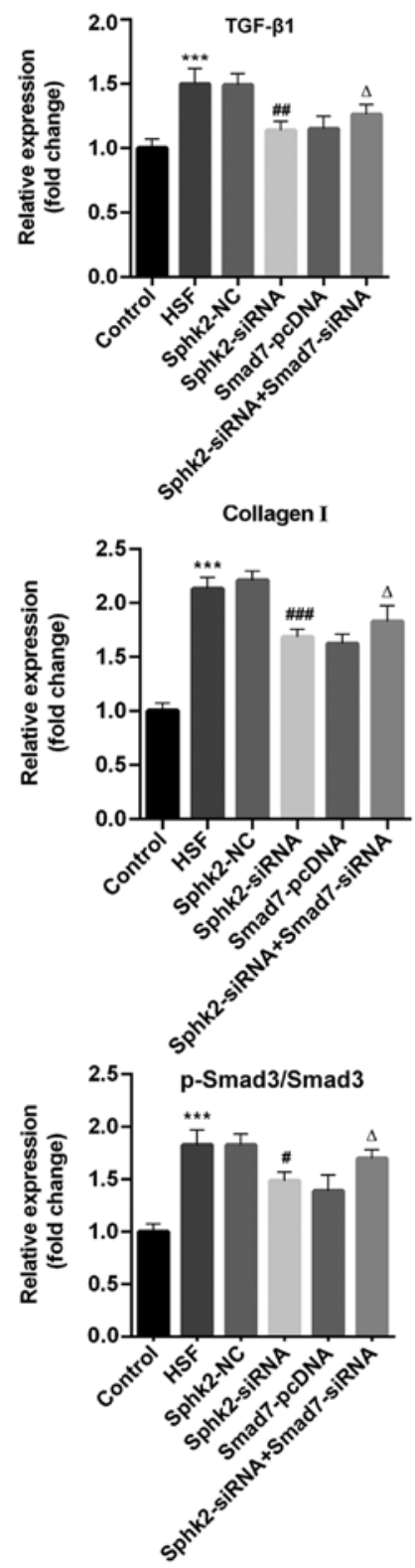

Figure 6. Effect of Sphk2-siRNA, Smad7-pcDNA and Sphk2-siRNA + Smad7-siRNA on the expression levels of TGF- $\beta 1 /$ Smad signaling key proteins and collagen I in HSF. Protein expression levels of (A) TGF- $\beta 1$ and (B) collagen I, p-Smad2 and p-Smad3 were determined using western blot analysis. ${ }^{* * * *} \mathrm{P}<0.001$ vs. the control; ${ }^{\#} \mathrm{P}<0.05,{ }^{\# \#} \mathrm{P}<0.01,{ }^{\# \# \#} \mathrm{P}<0.001$ vs. Sphk2-NC; ${ }^{\circ} \mathrm{P}<0.05$ vs. Sphk2-siRNA. All experiments were repeated three times independently. Data are presented as the mean \pm SD. Sphk2, sphingosine kinase 2 ; siRNA, small interfering RNA; TGF- $\beta 1$, transforming growth factor- $\beta 1$; NC, negative control; HSF, hypertrophic scar fibroblasts; p-, phosphorylated.

in the expression of the anti-apoptosis protein $\mathrm{Bcl}-2$ in the Sphk2-siRNA group or the Smad7-pcDNA group, which was eliminated by silencing of both Sphk2 and Smad7. Thus, it was demonstrated that Sphk2 silencing promoted apoptosis of HSF via upregulation of Smad7 expression.

It is speculated that an increase in collagen synthesis may be one of the main features of HS formation (32). Collagen I is the main structural element of the extracellular matrix, which plays as a vital role in the development and progression of HS (33). A previous study also reported that the expression of collagen I in HS tissues and HSF was notably higher compared with that in adjacent healthy skin tissues and normal cells, and the present results were in line with this previous study (34). Moreover, emerging evidence supports the hypothesis that Sphk2 could regulate the expression of collagen I in human corneal fibrosis (35). In addition, Sphk2 deficiency was revealed to decrease collagen accumulation in kidney tissues in a kidney fibrosis mouse model (13). The TGF- $\beta 1 /$ Smad signaling pathway plays a significant role during HS formation $(36,37)$. A previous study has revealed that activation of the TGF- $\beta 1 /$ Smad signaling pathway promoted HSF proliferation and collagen synthesis (38). Furthermore, Smad7, an inhibitor of Smads, is an essential negative regulator in the TGF- $\beta 1 /$ Smad signaling pathway (39), and it has been revealed that overexpression of Smad7 inhibits the fibrosis of hepatic stellate cells by regulating the TGF- $\beta 1 / \mathrm{Smad}$ signaling pathway (40). Moreover, Smad 7 acts as a negative feedback regulator, which can antagonize the activities of the Smad2 and Smad3 (41). In the present study, it was revealed that Sphk2 silencing or Smad7 overexpression decreased the expression levels of TGF- $\beta 1$, p-Smad2, p-Smad 3 and collagen I, which 
were reversed following transfection with both Sphk2- and Smad7-siRNA. Overall, the present results indicated that Sphk2 silencing inactivated TGF- $\beta 1 / \mathrm{Smad}$ signaling and collagen I expression in HSF by upregulating Smad7 expression.

In conclusion, to the best of our knowledge, the present study is the first to demonstrate that Sphk2 silencing may suppress HS formation via the inhibition of HSF proliferation, promotion of apoptosis, and inactivation of TGF- $\beta 1 / \mathrm{Smad}$ signaling and collagen I expression in HSF by upregulating Smad7 expression. Thus, Sphk2 may be a novel target for the treatment of HS. However, the fact that the specific relationship between Sphk2 and Smad7 was not determined is a limitation of the present research and therefore, a comprehensive analysis resolving these issues is required in the future.

\section{Acknowledgements}

Not applicable.

\section{Funding}

No funding was received.

\section{Availability of data and materials}

The analyzed data sets generated during the present study are available from the corresponding author on reasonable request.

\section{Authors' contributions}

$\mathrm{JZ}$ and BJ wrote the manuscript, interpreted the data and performed experiments. XX and RZ collected the data, searched the literature and designed the study. RZ revised the manuscript. All authors read and approval the final manuscript.

\section{Ethics approval and consent to participate}

The present study was approved by the Ethics Committee of The Second Affiliated Hospital, University of South China. Written informed consent was obtained from each patient or their legal guardians.

\section{Patient consent for publication}

Not applicable.

\section{Competing interests}

The authors declare that they have no competing interests.

\section{References}

1. Miletta NR, Donelan MB and Hivnor CM: Management of trauma and burn scars: The dermatologist's role in expanding patient access to care. Cutis 100: 18-20, 2017.

2. van der Veer WM, Bloemen MC, Ulrich MM, Molema G, van Zuijlen PP, Middelkoop E and Niessen FB: Potential cellular and molecular causes of hypertrophic scar formation. Burns 35: 15-29, 2009.

3. Sideek MA, Teia A, Kopecki Z, Cowin AJ and Gibson MA: Co-localization of LTBP-2 with FGF-2 in fibrotic human keloid and hypertrophic scar. J Mol Histol 47: 35-45, 2016.
4. Zuccaro J, Ziolkowski N and Fish J: A systematic review of the effectiveness of laser therapy for hypertrophic burn scars. Clin Plast Surg 44: 767-779, 2017.

5. Willows BM, Ilyas M and Sharma A: Laser in the management of burn scars. Burns 43: 1379-1389, 2017.

6. Gras C, Ratuszny D, Hadamitzky C, Zhang H, Blasczyk R and Figueiredo C: miR-145 contributes to hypertrophic scarring of the skin by inducing myofibroblast activity. Mol Med 21: 296-304, 2015

7. Wang XQ, Song F and Liu YK: Hypertrophic scar regression is linked to the occurrence of endothelial dysfunction. PLoS One 12: e0176681, 2017

8. Liu B, Guo Z and Gao W: miR-181b-5p promotes proliferation and inhibits apoptosis of hypertrophic scar fibroblasts through regulating the MEK/ERK/p21 pathway. Exp Ther Med 17: 1537-1544, 2019.

9. Pyne NJ, Dubois G and Pyne S: Role of sphingosine 1-phosphate and lysophosphatidic acid in fibrosis. Biochim Biophys Acta 1831: 228-238, 2013.

10. Schwalm S, Pfeilschifter J and Huwiler A: Sphingosine-1phosphate: A Janus-faced mediator of fibrotic diseases. Biochim Biophys Acta 1831: 239-250, 2013.

11. Ravichandran S, Finlin BS, Kern PA and Özcan S: Sphk2(-/-) mice are protected from obesity and insulin resistance. Biochim Biophys Acta Mol Basis Dis 1865: 570-576, 2019.

12. Schwalm S, Timcheva TM, Filipenko I, Ebadi M, Hofmann LP, Zangemeister-Wittke U, Pfeilschifter $\mathbf{J}$ and Huwiler A: Sphingosine kinase 2 deficiency increases proliferation and migration of renal mouse mesangial cells and fibroblasts. Biol Chem 396: 813-825, 2015.

13. Schwalm S, Beyer S, Frey H, Haceni R, Grammatikos G, Thomas D, Geisslinger G, Schaefer L, Huwiler A and Pfeilschifter J: Sphingosine kinase-2 deficiency ameliorates kidney fibrosis by up-regulating Smad7 in a mouse model of unilateral ureteral obstruction. Am J Pathol 187: 2413-2429, 2017.

14. Zhou J, Chen $\mathrm{J}$ and $\mathrm{Yu} \mathrm{H}$ : Targeting sphingosine kinase 2 by ABC294640 inhibits human skin squamous cell carcinoma cell growth. Biochem Biophys Res Commun 497: 535-542, 2018.

15. Shin SH, Cho KA, Hahn S, Lee Y, Kim YH, Woo SY, Ryu KH, Park WJ and Park JW: Inhibiting sphingosine kinase 2 derived-sphingosine-1-phosphate ameliorates psoriasis-like skin disease via blocking Th17 differentiation of naive CD4 T lymphocytes in mice. Acta Derm Venereol 99: 594-601, 2019.

16. Shi J, Li J, Guan H, Cai W, Bai X, Fang X, Hu X, Wang Y, Wang $\mathrm{H}$, Zheng Z, et al: Anti-fibrotic actions of interleukin-10 against hypertrophic scarring by activation of PI3K/AKT and STAT3 signaling pathways in scar-forming fibroblasts. PLoS One 9: e98228, 2014.

17. Zuo J, Chen Z, Zhong X, Lan W, Kuang Y and Huang D: FBP1 is highly expressed in human hypertrophic scars and increases fibroblast proliferation, apoptosis, and collagen expression. Connect Tissue Res 59: 120-128, 2018.

18. Chen H, Xu Y, Yang G, Zhang Q, Huang X, Yu L and Dong X: Mast cell chymase promotes hypertrophic scar fibroblast proliferation and collagen synthesis by activating TGF- $\beta 1 / \mathrm{Smads}$ signaling pathway. Exp Ther Med 14: 4438-4442, 2017.

19. Livak KJ and Schmittgen TD: Analysis of relative gene expression data using real-time quantitative PCR and the 2(-Delta Delta C(T)) method. Methods 25: 402-408, 2001.

20. Xiao Y, Xu D, Song H, Shu F, Wei P, Yang X, Zhong C, Wang X, Müller WE, Zheng Y, et al: Cuprous oxide nanoparticles reduces hypertrophic scarring by inducing fibroblast apoptosis. Int J Nanomedicine 14: 5989-6000, 2019.

21. Zhou Y, Zhao Y, Du H, Suo Y, Chen H, Li H, Liang X, Li Q and Huang X: Downregulation of CFTR is involved in the formation of hypertrophic scars. Biomed Res Int 2020: 9526289 , 2020.

22. Song Y, Guo B, Ma S, Chang P and Tao K: Naringin suppresses the growth and motility of hypertrophic scar fibroblasts by inhibiting the kinase activity of Akt. Biomed Pharmacother 105: 1291-1298, 2018.

23. Zhou X, Xie Y, Xiao H, Deng X, Wang Y, Jiang L, Liu C and Zhou R: MicroRNA-519d inhibits proliferation and induces apoptosis of human hypertrophic scar fibroblasts through targeting Sirtuin 7. Biomed Pharmacother 100: 184-190, 2018.

24. Qiu W, Yang Z, Fan Y and Zheng Q: MicroRNA-613 inhibits cell growth, migration and invasion of papillary thyroid carcinoma by regulating SphK2. Oncotarget 7: 39907-39915, 2016. 
25. Zhang L, Liu X, Zuo Z, Hao C and Ma Y: Sphingosine kinase 2 promotes colorectal cancer cell proliferation and invasion by enhancing MYC expression. Tumor Biol 37: 8455-8460, 2016.

26. Leili H, Nasser S, Nadereh R, Siavoush D and Pouran K: Sphingosine kinase-2 inhibitor ABC294640 enhances doxorubicin-induced apoptosis of NSCLC cells via altering survivin expression. Drug Res (Stuttg) 68: 45-53, 2018.

27. Xiao Y, Liu R, Li X, Gurley EC, Hylemon PB, Lu Y, Zhou H and Cai W: Long noncoding RNA H19 contributes to cholangiocyte proliferation and cholestatic liver fibrosis in biliary atresia. Hepatology 70: 1658-1673, 2019.

28. Wang XC, Wang T, Zhang Y, Wang LL, Zhao RY and Tan W: Tacrolimus inhibits proliferation and induces apoptosis by decreasing survivin in scar fibroblasts after glaucoma surgery Eur Rev Med Pharmacol Sci 22: 2934-2940, 2018.

29. Li XY, Li T, Li XJ, Wang JN and Chen Z: TSG-6 induces apoptosis of human hypertrophic scar fibroblasts via activation of the Fas/FasL signalling pathway. Folia Biol (Praha) 64: 173-181, 2018.

30. Zhang G, Zheng H, Zhang G, Cheng R, Lu C, Guo Y and Zhao G: MicroRNA-338-3p suppresses cell proliferation and induces apoptosis of non-small-cell lung cancer by targeting sphingosine kinase 2. Cancer Cell Int 17: 46, 2017.

31. Fan X, Yuan J, Xie J, Pan Z, Yao X, Sun X, Zhang P and Zhang L: Long non-protein coding RNA DANCR functions as a competing endogenous RNA to regulate osteoarthritis progression via miR-577/SphK2 axis. Biochem Biophys Res Commun 500: 658-664, 2018.

32. Ma L, Li LY and Zhao TL: Anti-inflammatory effects of ginsenoside $\operatorname{Rg} 3$ on the hypertrophic scar formation via the NF- $\mathrm{kB} / \mathrm{I} \kappa \mathrm{B}$ signaling pathway in rabbit ears. Pharmazie 75: 102-106, 2020.

33. Volk SW, Wang Y, Mauldin EA, Liechty KW and Adams SL: Diminished type III collagen promotes myofibroblast differentiation and increases scar deposition in cutaneous wound healing. Cells Tissues Organs 194: 25-37, 2011.

34. Zhou R, Zhang Q, Zhang Y, Fu S and Wang C: Aberrant miR-21 and miR-200b expression and its pro-fibrotic potential in hypertrophic scars. Exp Cell Res 339: 360-366, 2015.
35. Nicholas SE, Rowsey TG, Priyadarsini S, Mandal NA and Karamichos D: Unravelling the interplay of sphingolipids and TGF- $\beta$ signaling in the human corneal stroma. PLoS One 12: e0182390, 2017.

36. Zhang Y, Shan S, Wang J, Cheng X, Yi B, Zhou J and Li Q: Galangin inhibits hypertrophic scar formation via ALK5/Smad2/3 signaling pathway. Mol Cell Biochem 413: 109-118, 2016.

37. Zhao JC, Zhang BR, Shi K, Wang J, Yu QH and Yu JA: Lower energy radial shock wave therapy improves characteristics of hypertrophic scar in a rabbit ear model. Exp Ther Med 15: 933-939, 2018.

38. Chen H, Xu Y, Yang G, Zhang Q, Huang X, Yu L and Dong X: Mast cell chymase promotes hypertrophic scar fibroblast proliferation and collagen synthesis by activating TGF- $\beta 1 /$ Smads signaling pathway. Exp Ther Med 14: 4438-4442, 2017.

39. Seki N, Toh U, Kawaguchi K, Ninomiya M, Koketsu M, Watanabe K, Aoki M, Fujii T, Nakamura A, Akagi Y, et al: Tricin inhibits proliferation of human hepatic stellate cells in vitro by blocking tyrosine phosphorylation of PDGF receptor and its signaling pathways. J Cell Biochem 113: 2346-2355, 2012.

40. Wu SP, Yang Z, Li FR, Liu XD, Chen HT and Su DN: Smad7-overexpressing rat BMSCs inhibit the fibrosis of hepatic stellate cells by regulating the TGF- $\beta 1 /$ Smad signaling pathway. Exp Ther Med 14: 2568-2576, 2017.

41. Tang B, Zhu B, Liang Y, Bi L, Hu Z, Chen B, Zhang K and Zhu J: Asiaticoside suppresses collagen expression and TGF- $\beta / \mathrm{Smad}$ signaling through inducing Smad7 and inhibiting TGF- $\beta$ RI and TGF- $\beta$ RII in keloid fibroblasts. Arch Dermatol Res 303: 563-572, 2011.

This work is licensed under a Creative Commons Attribution-NonCommercial-NoDerivatives 4.0 International (CC BY-NC-ND 4.0) License. 